\title{
Solution to pink tea challenge
}

\author{
Lucia D'Ulivo ${ }^{1}$
}

(C) Her Majesty the Queen in Right of Canada as represented by: NRC Canada 2017

The winner of the pink tea challenge (published in volume 409 issue 17) is:

Beatrice Campanella, Italian National Research Council (CNR)-Institute of chemistry of Organometallic Compounds (ICCOM), Pisa, Italy.

The award entitles the winner to select a Springer book of her choice up to a value of $€ 100,-$.

Our Congratulations!

\section{Solution}

Pink tea, also known as Noon chai, is a traditional Kashmiri beverage prepared with special tea leaves, such as the Kashmiri chai or green tea, water, salt, baking soda, and milk.

Tea leaves contain hundreds of different types of polyphenols [1, 2], which may become protonated at acidic $\mathrm{pH}$ or ionized in alkaline conditions [3]. Therefore, polyphenols and, consequently, the tea can act as natural acid-base indicators [3]. Specifically, polyphenols in green or Kashmiri chai act similar to phenolsulfonphthalein, a common $\mathrm{pH}$ indicator better known as phenol red, which is yellow at acidic $\mathrm{pH}$ and red in alkaline conditions (Fig. 1).

This article is the solution to the Analytical Challenge to be found at http://dx.doi.org/10.1007/s00216-017-0340-8

Lucia D'Ulivo

lucia.dulivo@gmail.com

1 National Research Council of Canada, 1200 Montreal Road, Ottawa, ON K1A 0R6, Canada
During the preparation of Noon chai, tea leaves are boiled for a long time (about an hour) to maximize the extraction of polyphenols [4]. Using tea bags is not recommended since it can decrease the extraction efficiency of polyphenols [4]. By adding baking soda, the tea extract assumes a red color, which turns pink with the final addition of milk. In the case of Kashmiri chai blend or green tea, the extract turns from a pale-brown yellow to a dark-brown red color as the $\mathrm{pH}$ becomes alkaline. This is why adding baking soda is essential for obtaining the characteristic pink color.

For the preparation of pink tea, Kashmiri chai blend or green tea works the best since their content in polyphenols is higher compared to other teas [4]. On the other hand, white tea has a relative low content of polyphenols [4]. Indeed, a white tea infusion looks pale-yellow and turns light brown by adding baking soda. Although a change in coloration is observable, it is not quite enough to obtain the fancy pink tea.

Many herbal teas, such as hibiscus flowers, contain anthocyanins - a class of polyphenolic compounds that also act as $\mathrm{pH}$ indicators. In the case of hibiscus flowers, the acid and base colors are red and green, respectively [3]. A similar phenomenon occurs with other herbal teas such as Red Zinger or Mandarin Orange [3]. In this case, the addition

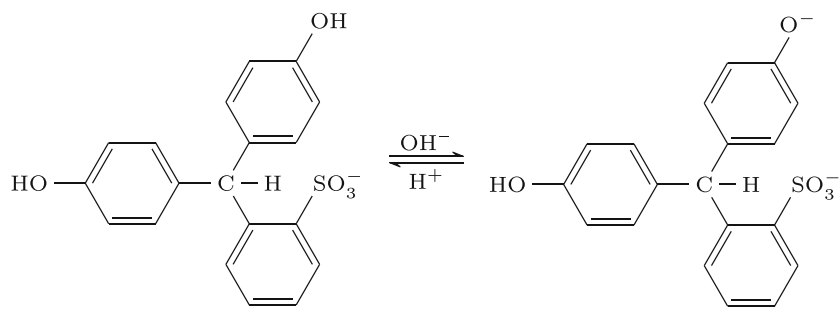

Fig. 1 Acid-base equilibrium of phenolsulfonphthalein (Phenol Red). Phenolsulfonphthalein is yellow in acidic conditions and turns red in alkali 
of baking soda would yield a "green" tea extract, instead of pink.

\section{References}

1. Kuhnert N. Unraveling the structure of the black tea thearubigins. Arch Biochem Biophys. 2010;501:37-51.
2. Menet M-C, Sang S, Yang CS, Ho C-T, Rosen RT. Analysis of theaflavins and thearubigins from black tea extract by MALDI-TOF mass spectrometry. J Agric Food Chem. 2004;52: 2455-61.

3. Epp DN. Teas as natural indicators. J Chem Educ. 1993;70: 326.

4. Rusak G, Komes D, Likić S, Horžić D, Kovać M. Phenolic content and antioxidative capacity of green and white tea extracts depending on extraction conditions and the solvent used. Food Chem. 2008;110:852-8. 